International Journal of Recent advances in Physics (IJRAP) Vol.4, No.3, August 2015

\title{
EXACT SOLUTIONS OF SCHRÖDINGER EQUATION WITH SOLVABLE POTENTIALS FOR NON PT/PT-SYMMETRIC
}

\author{
Akaninyene D. Antia*, Imeh E. Essien and Eno E. Ituen \\ Theoretical Physics Group, Department of Physics, University of Uyo, Nigeria.
}

\begin{abstract}
We have obtained explicitly the exact solutions of the Schrodinger equation with Non PT/PT symmetric Rosen Morse II, Scarf II and Coulomb potentials. Energy eigenvalues and the corresponding unnormalized wave functions for these systems for both Non PT and PT symmetric are also obtained using the Nikiforov-Uvarov (NU) method.
\end{abstract}

\section{KEYWORDS}

Non PT/PT symmetry, Nikiforov-Uvarov method, eigenvalues and wave functions.

\section{INTRODUCTION}

In different areas of physics we are bombarded with various types of potentials ( real or complex forms) and the past decade has seen the renaissance of non-Hermitian quantum mechanical systems after the introduction of PT-symmetric quantum system [1-4]. Non Hermitian complex Hamiltonian whose energy levels are real and positive as a result of PT-symmetry is the building block for an Hermitian Hamiltonians aspect of a physical theory of quantum mechanics [1,4]. Hamiltonian $H$ for a PT-symmetric must satisfies $[P T, H]=0$, this means that $P T H(P T)^{-1}=H$, where $\mathrm{P}$ and $\mathrm{T}$ are the space and time-reversal transformations respectively $[1,4]$. The $\mathrm{P}$ and $\mathrm{T}$ obey the following definitions: $P x P=-x, P p P=T p T=-p$ and TiIT $=-i I$ where $x, p$ and $I$ are the position, momentum and identity operators respectively acting on the Hilbert space $H=L^{2}(R)$ and $i=\sqrt{-1}[1,4]$. It should be noted that the above definition is only applicable to a system with real $x$ and $p[1,5]$.

The recent symmetry concept, the PT-symmetry of 1-D quantum potentials in quantum mechanics is invariance under spontaneous $P$ and $T$ reflection [6,7]. Obtaining exact solutions forpotential with complex spectrum under Schrödinger equation is of great interest[7]. It must be stated that PT-symmetry does not actually bring about real spectrum. If PT-symmetry is not broken spontaneously then Hamiltonian spectrum will be real and the PT symmetry quantum mechanics has been identified as special case of pseudo-Hermiticity [5]. Actually, Hamiltonian of this nature is known as pseudo Hamiltonian if $H^{+}=\eta H \eta^{-1}$, where + represents operator of adjunct [8]. Also a new class of non-Hermitian Hamiltonians with real spectra which are obtained using pseudo-symmetry had been proposed [9].

In studying PT-invariant potentials various techniques have been applied such as numerical approaches [10], semi-classical estimates [11], quantum field theory [12], variation methods [13],

DOI : 10.14810/ijrap.2015.4302 
Lie group theoretical approaches [14]. Recently an alternative method for solving Schrödinger, Dirac and Klein Gordon equation has been introduced called Nikiforov-Uvarov (NU) method [15].

In this work, main aim is to obtain the energy eigenvalues and corresponding eigenfunction of the solvable potentials both nonPT and PT-symmetric under the frame work of NU-method.The paper is arranged as follows: In section 2, the Nikiforov-Uvarov (NU) method is briefly introduced. In section 3, solutions of Rosen-Morse II potential Scarf II potential and coulomb potential are presented. In section 4, PT symmetric solutions of the solvable potentials: Rosen Morse, Scarf II, and Coulomb potentials are presented. Conclusion is presented in section 5.

\section{A BRIEF REVIEW OF NIKIFOROV-UVAROV (NU) METHOD}

One of the recently introduced methods for solving second order differential equations such as Schrodinger, Klein-Gordon, Dirac equations, etc. is the NU method[15]. It is based on solving the second order linear differential equation by reducing to a generalized equation of hypergeometric type [16]. Under NU method, the second order linear differential equations is written as follows,

$$
\psi^{\prime \prime}(s)+\frac{\bar{\tau}(s)}{\sigma(s)} \psi^{\prime}(s)+\frac{\tilde{\sigma}}{\sigma^{2}(s)} \psi(s)=0,
$$

where $\sigma(s)$ and $\tilde{\sigma}(s)$ are polynomials, at most second order, and $\bar{\tau}(s)$ is a first order polynomial[15]. The particular solution of $\mathrm{Eq}(1)$ can be obtained by using the following transformation,

$$
\psi(s)=\phi(s) y(s)
$$

It reduces $\mathrm{Eq}(1)$ to hypergeometric-type equation of the form

$$
\sigma(s) y+\tau(s) y+\lambda y=0
$$

where $\phi(s)$ must satisfies the equation [15]

$$
\frac{\phi^{1}(s)}{\phi(s)}=\frac{\pi(s)}{\sigma(s)}
$$

The wave function $y(s)$ is the hypergeometric-type function whose polynomial solution is obtained using the Rodriquez relation as[15]

$$
y_{n}(s)=\frac{B_{n}}{\rho(s)} \frac{d^{n}}{d s^{n}}\left[\sigma^{n} \rho(s)\right]
$$

where $B_{n}$ is the normalization constant . The following condition must be satisfied by the weight function $\rho(s)$ : 


$$
\frac{d}{d s}(\sigma(s) \rho(s))=\tau(s) \rho(s)
$$

For NU method, the function $\pi(s)$ and the parameter $\lambda$ are defined respectfully as follows

$$
\begin{aligned}
& \pi(s)=\frac{\sigma^{\prime}-\bar{\tau}}{2} \pm \sqrt{\left(\frac{\sigma^{\prime}-\bar{\tau}}{2}\right)^{2}}-\tilde{\sigma}+k \sigma \\
& \lambda=k+\pi^{\prime}(s)
\end{aligned}
$$

However, to obtain the value of $k$, the expression under the square root must be the square of the polynomial. This implies that another eigenvalue equation for the second order equation becomes

$$
\lambda=\lambda_{n}=-n \tau^{\prime}-\frac{n(n-1)}{2} \sigma^{\prime \prime}(s)
$$

where

$$
\tau(s)=\bar{\tau}(s)+2 \pi(s)
$$

and its derivative must be negative. If we compare Eqs (8) and (9), the energy eigenvalues is obtained

\section{EXACT SOLUTIONS OF SCHRÖDINGER EQUATION WITH SOLVABLE POTENTIALS}

In this section we will obtain the solutions of three exactly solvable potentials: Rosen-Morse II, Scarf II and Coulomb potentials using NU method

\subsection{Solutions of Rosen-Morse II Potential}

The Rosen-Morse II potential is defined as [3]

$$
V(x)=\frac{a(a+1)}{\cos h^{2}(\alpha x)}+2 \gamma \tanh (\alpha x)
$$

where $a, \alpha$ and $\gamma$ are adjustable parameters. The Schrödinger equation in one dimension is given as

$$
\frac{d^{2} \psi(x)}{d x^{2}}+\frac{2 \mu}{\hbar^{2}}[E-V(x)] \psi(x)=0
$$

Substituting Eq. (11) into Eq. (12), we obtain

$$
\frac{d^{2} \psi(x)}{d x^{2}}+\frac{2 \mu}{\hbar^{2}}\left[E+\frac{a(a+1)}{\cos h^{2}(\alpha x)}-2 \gamma \tanh (\alpha x)\right] \psi(x)=0
$$

By introducing a new transformation $s=\tanh (\alpha x)$ in Eq. (13), we have 
International Journal of Recent advances in Physics (IJRAP) Vol.4, No.3, August 2015

$$
\frac{d^{2} \psi(s)}{d s^{2}}-\frac{2 s}{\left(1-s^{2}\right)} \frac{d \psi(s)}{d s}+\frac{1}{\left(1-s^{2}\right)^{2}}\left[-\delta^{2} s^{2}-\Lambda^{2} s+\left(\delta^{2}-\varepsilon^{2}\right)\right] \psi(s)=0
$$

In obtaining Eq. (14) the following dimensionless quantities have been used:

$$
-\varepsilon^{2}=\frac{2 \mu \mathrm{E}}{\alpha^{2} \hbar^{2}}, \delta^{2}=\frac{2 \mu a(a+1)}{\alpha^{2} \hbar^{2}}, \Lambda^{2}=\frac{4 \mu \gamma}{\alpha^{2} \hbar^{2}}
$$

Comparing Eq. (14) with Eq. (1), we obtain the following polynomials

$$
\tilde{\tau}=-2 s, \sigma(s)=1-s^{2}, \tilde{\sigma}=-\delta^{2} s^{2}-\Lambda^{2} s+\left(\delta^{2}-\varepsilon^{2}\right)
$$

The function $\pi(s)$ can be obtained using Eqs (16) and (7) as

$$
\pi(s)= \pm \sqrt{\left(\delta^{2}-k\right) s^{2}+\Lambda^{2} s+\left(\varepsilon^{2}+k-\delta^{2}\right)}
$$

The expression in the square root, based on NU method, must be square of a polynomial, thus the constant $k$ can be determined as

$$
k=\delta^{2}-\frac{\varepsilon^{2}}{2} \pm \frac{1}{2} \sqrt{\left(\varepsilon^{2}-\Lambda^{2}\right)\left(\varepsilon^{2}+\Lambda^{2}\right)}
$$

At this point, the function $\pi(s)$ for each value of $k$ is given as

$$
\pi(s)= \pm\left\{\begin{array}{c}
\frac{1}{2}\left(\sqrt{\left(\varepsilon^{2}-\Lambda^{2}\right)}-\sqrt{\left(\varepsilon^{2}+\Lambda^{2}\right)}\right) s-\frac{1}{2}\left(\sqrt{\left(\varepsilon^{2}-\Lambda^{2}\right)}+\sqrt{\left(\varepsilon^{2}+\Lambda^{2}\right)}\right) \\
\text { for } k=\delta^{2}-\frac{\varepsilon^{2}}{2}+\frac{1}{2} \sqrt{\left(\varepsilon^{2}-\Lambda^{2}\right)\left(\varepsilon^{2}+\Lambda^{2}\right)} \\
\frac{1}{2}\left(\sqrt{\left(\varepsilon^{2}-\Lambda^{2}\right)}+\sqrt{\left(\varepsilon^{2}+\Lambda^{2}\right)}\right) s-\left(\sqrt{\left(\varepsilon^{2}-\Lambda^{2}\right)}-\sqrt{\left(\varepsilon^{2}+\Lambda^{2}\right)}\right) \\
\text { for } k=\delta^{2}-\frac{\varepsilon^{2}}{2}-\frac{1}{2} \sqrt{\left(\varepsilon^{2}-\Lambda^{2}\right)\left(\varepsilon^{2}+\Lambda^{2}\right)}
\end{array}\right.
$$

The negative derivative of the relation, $\tau(s)=\bar{\tau}(s)+2 \pi(s)$, can be obtained if we select

$$
\begin{aligned}
\pi(s) & =-\frac{1}{2}\left(\sqrt{\left(\varepsilon^{2}-\Lambda^{2}\right)}-\sqrt{\left(\varepsilon^{2}+\Lambda^{2}\right)}\right) s+\frac{1}{2}\left(\sqrt{\left(\varepsilon^{2}-\Lambda^{2}\right)}-\sqrt{\left(\varepsilon^{2}+\Lambda^{2}\right)}\right) \\
k & =\delta^{2}-\frac{\varepsilon^{2}}{2}-\frac{1}{2} \sqrt{\left(\varepsilon^{2}-\Lambda^{2}\right)\left(\varepsilon^{2}+\Lambda^{2}\right)}
\end{aligned}
$$

With this selection, we obtain $\tau(s)$ as

$$
\tau(s)=-2 s-\left(\sqrt{\left(\varepsilon^{2}-\Lambda^{2}\right)}+\sqrt{\left(\varepsilon^{2}+\Lambda^{2}\right)}\right) s+\left(\sqrt{\left(\varepsilon^{2}-\Lambda^{2}\right)}-\sqrt{\left(\varepsilon^{2}+\Lambda^{2}\right)}\right)
$$


$\tau^{1}(s)=-\left(2+\sqrt{\left(\varepsilon^{2}-\Lambda^{2}\right)}+\sqrt{\left(\varepsilon^{2}+\Lambda^{2}\right)}\right)$

Using Eq. (8), who obtain $\lambda$ as

$$
\lambda=\delta^{2}-\frac{\varepsilon^{2}}{2}-\frac{1}{2} \sqrt{\left(\varepsilon^{2}-\Lambda^{2}\right)\left(\varepsilon^{2}+\Lambda^{2}\right)}-\frac{1}{2}\left(\sqrt{\left(\varepsilon^{2}-\Lambda^{2}\right)}+\sqrt{\left(\varepsilon^{2}+\Lambda^{2}\right)}\right)
$$

Also, using Eq. (9), we have

$$
\lambda=\lambda_{n}=n\left(2+\sqrt{\varepsilon^{2}-\Lambda^{2}}+\sqrt{\varepsilon^{2}+\Lambda^{2}}\right)+n(n+1)
$$

Solving Eqs (23) and (24) and making use of Eq (15), we obtain the energy eigenvalues of the system as

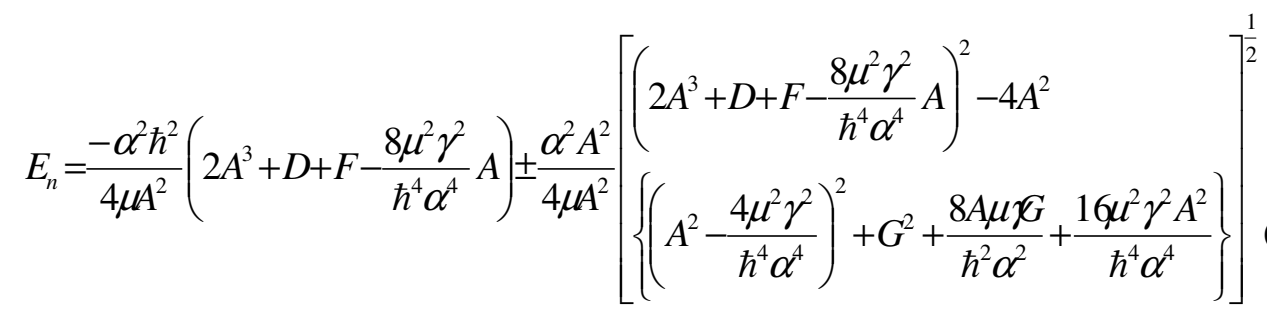

where,

$$
\begin{aligned}
& A=\frac{2 \mu a(a+1)}{\hbar^{2} \alpha^{2}}-2 n-n(n-1) \\
& D=\left[\left(\frac{4 \mu a(a+1)}{\hbar^{2} \alpha^{2}}-4 n-2 n(n-1)\right)^{2}-\frac{16 \mu^{2} \gamma^{2}}{\hbar^{2} \alpha^{2}}\right]\left(n+\frac{1}{2}\right)^{2} \\
& F=\frac{16 \mu^{2} \gamma^{2}}{\hbar^{4} \alpha^{4}}\left(\frac{2 \mu a(a+1)}{\hbar^{2} \alpha^{2}}+n(n-1)+\frac{1}{2}\right) \\
& G=\frac{8 \mu \gamma}{\hbar^{2} \alpha^{2}}\left(n+\frac{1}{2}\right)^{2}
\end{aligned}
$$

Using Eq. (6), the weight function $\rho(s)$ can be found to be

$$
\rho(s)=(1+s)^{\xi+v}
$$

where $\xi=\sqrt{\varepsilon^{2}-\Lambda^{2}}$ and $v=\sqrt{\varepsilon^{2}+\Lambda^{2}}$

Substituting Eq. (26) into Eq. (5), we obtain $y_{n}(s)$ as 


$$
y_{n}(s)=B_{n}(1+s)^{-(\xi+v)} \frac{d^{n}}{d s^{n}}\left[(1-s)^{n}(1+s)^{n+(\xi+v)}\right]
$$

The other wave function $\phi(s)$ can be obtained using Eq. (4) as

$$
\phi(s)=(1-s)^{\frac{v}{2}}(1+s)^{\frac{\xi}{2}}
$$

Also using $\psi(s)=\phi(s) y(s)$, the solution of Eq (14) can be written as

$$
\psi(s)=B_{n}(1-\tanh (\alpha x))^{\frac{v}{2}}(1+\tanh (\alpha x))^{\frac{\xi}{2}} P_{n}^{(\xi+v)}(\tanh (\alpha x)),
$$

where $B_{n}$ is the normalization constant and $P_{n}^{(\xi+v)}$ is the Jacobi polynomials.

\subsection{Solutions of Scarf II Potential}

The scarf II potential is given as [3]

$$
V(x)=\frac{-1}{\cosh ^{2} \alpha x}\left[\left(\frac{\gamma+\beta}{2}\right)^{2}+\left(\frac{\gamma-\beta}{2}\right)^{2}-\frac{1}{4}\right]+\left(\frac{\beta+\gamma}{2}\right)\left(\frac{\beta-\gamma}{2}\right) \frac{2 \sin h \alpha x}{\cos h^{2} \alpha x},
$$

where $\beta$ is a parameter.

Substituting Eq. (30) in Eq. (12) and using the transformation, $s=\sinh (\alpha x$, we have

$$
\frac{d^{2} \psi}{d s^{2}}+\frac{s}{\left(1+s^{2}\right)} \frac{d \psi}{d s}+\frac{s}{\left(1+s^{2}\right)^{2}}\left[-\varepsilon^{2} s^{2}-2 \delta^{2} s-\left(\varepsilon^{2}-g^{2}\right)\right] \psi(s)=0,
$$

In obtaining Eq. (31) the following dimensionless quantities have been used:

$$
-\varepsilon^{2}=\frac{2 \mu E}{\alpha^{2} \hbar^{2}}, \delta^{2}=\frac{2 \mu}{\alpha^{2} \hbar^{2}}\left(\frac{\gamma^{2}}{2}+\frac{\beta^{2}}{2}-\frac{1}{4}\right) \text { and } g^{2}=\frac{\mu}{2 \alpha^{2} \hbar^{2}}\left(\beta^{2}-\gamma^{2}\right)
$$

Comparing $\mathrm{Eq}(31)$ with $\mathrm{Eq}(1)$, we obtain

$$
\tilde{\tau}(s)=s, \sigma(s)=1+s^{2}, \tilde{\sigma}(s)=-\varepsilon^{2} s^{2}-2 \delta^{2} s-\left(\varepsilon^{2}-g^{2}\right)
$$

Using Eqs. (7) and (33), we get the function $\pi(s)$ as

$$
\pi(s)=\frac{s}{2} \pm \sqrt{\left(\varepsilon^{2}+k+\frac{1}{4}\right) s^{2}+2 \delta^{2} s+\left(\varepsilon^{2}-g^{2}+k\right)}
$$

Based on NU method, the expression in the square root in Eq.(34) must be a polynomial and with this the constant $k$ can be determined as 


$$
k=\frac{-8 \varepsilon^{2}+4 g^{2}-1 \pm \sqrt{\left(4 g^{2}+1\right)^{2}+64 \delta^{4}}}{8}
$$

For each value of $k$, the function $\pi(s)$ can be found as

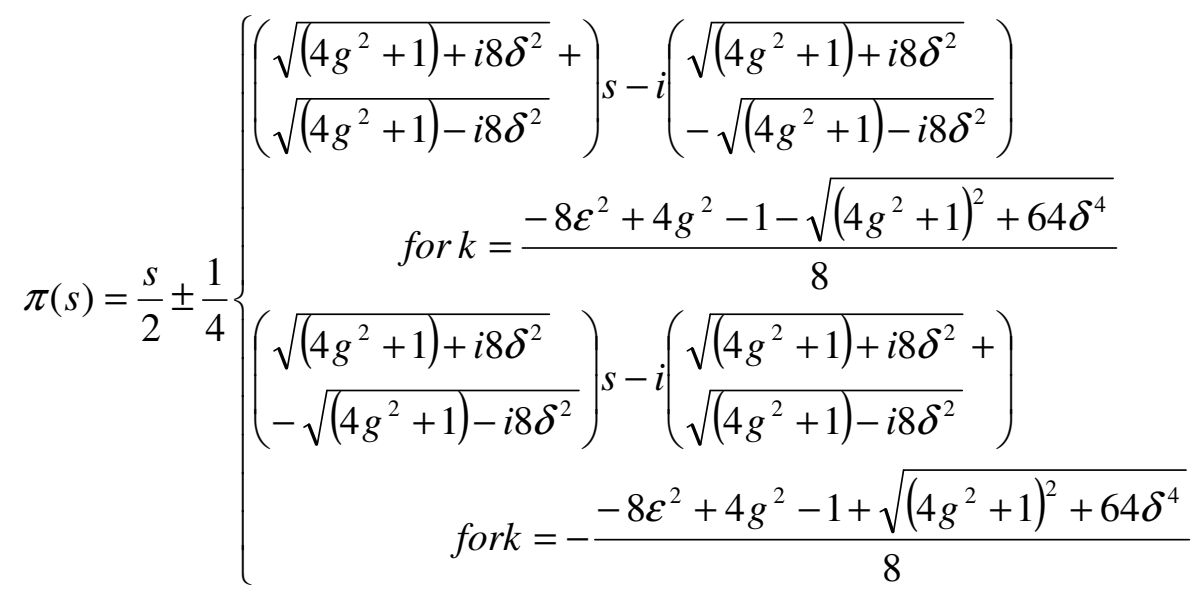

Appropriate value of $\pi(s)$ is chosen such that the function $\tau(s)$ in Eq. (10) will have negative derivative [16]. So we select the polynomial values to be

$$
\pi(s)=\frac{s}{2}-\frac{1}{4}\left(\begin{array}{l}
\sqrt{\left(4 g^{2}+1\right)+i 8 \delta^{2}}- \\
\sqrt{\left(4 g^{2}+1\right)-i 8 \delta^{2}}
\end{array}\right) s+\frac{1}{4}\left(\begin{array}{l}
\sqrt{\left(4 g^{2}+1\right)+i 8 \delta^{2}}+ \\
\sqrt{\left(4 g^{2}+1\right)-i 8 \delta^{2}}
\end{array}\right)
$$

and

$$
k=\frac{-8 \varepsilon^{2}+4 g^{2}-1-\sqrt{\left(4 g^{2}+1\right)^{2}+64 \delta^{4}}}{8}
$$

Which yields

$$
\begin{aligned}
& \tau(s)=2 s-\frac{1}{2}\left[\left(\begin{array}{l}
\sqrt{\left(4 g^{2}+1\right)+i 8 \delta^{2}}- \\
\sqrt{\left(4 g^{2}+1\right)-i 8 \delta^{2}}
\end{array}\right) s-i\left(\begin{array}{l}
\sqrt{\left(4 g^{2}+1\right)+i 8 \delta^{2}}+ \\
\sqrt{\left(4 g^{2}+1\right)-i 8 \delta^{2}}
\end{array}\right)\right] \\
& \tau^{\prime}(s)=-\left[\frac{1}{2}\left(\sqrt{\left(4 g^{2}+1\right)+i 8 \delta^{2}}-\sqrt{\left.\left(4 g^{2}+1\right)-i 8 \delta^{2}\right)}-2\right]\right.
\end{aligned}
$$

Using Eqs. (8) and (9) the following expression for $\lambda$ are obtained, respectively,

$$
\begin{array}{r}
\lambda=\frac{1}{2}-\frac{1}{4}\left(\begin{array}{c}
\sqrt{\left(4 g^{2}+1\right)+i 8 \delta^{2}}- \\
\sqrt{\left(4 g^{2}+1\right)-i 8 \delta^{2}}
\end{array}\right)+\frac{-8 \varepsilon^{2}+4 g^{2}-1-\sqrt{\left(4 g^{2}+1\right)^{2}+64 \delta^{4}}}{8} \\
\lambda=\lambda_{n}=\left[\frac{1}{2}\left(\sqrt{\left(4 g^{2}+1\right)+i 8 \delta^{2}}-\sqrt{\left(4 g^{2}+1\right)-i 8 \delta^{2}}\right)-2\right] n-n(n-1)
\end{array}
$$


Combining Eqs. (40) and (41) the energy eigenvalues of this system is obtained as

$$
E_{n}=-\frac{1}{2}\left(\beta^{2}-\gamma^{2}\right)+\frac{\alpha^{2} \hbar^{2}}{2 \mu}\left[\begin{array}{l}
\frac{1}{8} \sqrt{\left(1+\frac{2 \mu}{\alpha^{2} \hbar^{2}}\left(\beta^{2}-\gamma^{2}\right)\right)^{2}+\frac{64 \mu^{2}}{\alpha^{2} \hbar^{2}}\left(\gamma^{2}+\beta^{2}-\frac{1}{2}\right)^{2}}-\frac{3}{8}-2 n-n(n-1)+ \\
\left(\frac{1+2 n}{4}\right) \\
\sqrt{\sqrt{1+\frac{2 \mu}{\alpha^{2} \hbar^{2}}\left(\beta^{2}-\gamma^{2}\right)+\frac{i 8}{\alpha^{2} \hbar^{2}}\left(\gamma^{2}+\beta^{2}-\frac{1}{2}\right)}} \\
\sqrt{1+\frac{2 \mu}{\alpha^{2} \hbar^{2}}\left(\beta^{2}-\gamma^{2}\right)-\frac{i 8}{\alpha^{2} \hbar^{2}}\left(\gamma^{2}+\beta^{2}-\frac{1}{2}\right)}
\end{array}\right]
$$

By using Eq. (6) the weight function $\rho(s)$ is obtained as

$$
\rho(s)=(1+i s)^{\frac{1}{2} \xi^{\prime}}(1-i s)^{-\frac{1}{2} v^{\prime}}
$$

where

$$
\begin{aligned}
& v^{\prime}=\sqrt{\left(4 g^{2}+1\right)-i 8 \delta^{2}} \\
& \xi^{\prime}=\sqrt{\left(4 g^{2}+1\right)+i 8 \delta^{2}}
\end{aligned}
$$

Substituting Eq. (43) into Eq. (5), we obtain

$$
y_{n}(s)=N_{n}(1+i s)^{-\frac{1}{2} \xi^{\prime}}(1-i s)^{\frac{1}{2} v^{\prime}} \frac{d^{n}}{d s^{n}}\left[(1+i s)^{n+\frac{1}{2} \xi^{\prime}}(1-i s)^{n-\frac{1}{2} v^{\prime}}\right]
$$

From $\mathrm{Eq}(4)$, other wave function $\varphi(s)$ is obtained as

$\varphi(s)=(1+i s)^{\frac{1}{4}\left(1+\xi^{\prime}\right)}(1-i s)^{\frac{1}{4}\left(1-v^{\prime}\right)}$

Combining Eqs(44) and (45), the wave function for this system is

$\psi(x)=N_{n}(1+i \sinh (\alpha x))^{\frac{1}{4}\left(1+\xi^{\prime}\right)}(1-i \sinh (\alpha x))^{\frac{1}{4}\left(1-v^{\prime}\right)} \times P_{n}^{\left(\frac{1}{2} \xi^{\prime \prime},-\frac{1}{2} v^{\prime}\right)}(i \sinh (\alpha x))$

where normalization constant is $N_{n}$ and $P_{n}^{\left(\frac{1}{2} \xi^{\prime},-\frac{1}{2} v^{\prime}\right)}$ is Jacobi polynomial.

\subsection{Solutions of Coulomb potential}

The coulomb potential is defined as [3]

$$
V(x)=\frac{z}{\alpha x}+\frac{l(1+l)}{\alpha x^{2}},
$$

Where $\mathrm{Z}$ is the atomic number. Subsisting Eq. (47) into Eq. (12) yield

$$
\frac{d^{2} \psi(x)}{d x^{2}}+\frac{1}{x^{2}}\left[-\varepsilon^{2} x^{2}-\delta^{2} x-\Lambda^{2}\right] \psi(x)=0
$$


The dimensionless quantities used in obtaining Eq. (48) are

$$
-\varepsilon^{2}=\frac{2 \mu E}{\hbar^{2}}, \delta^{2}=\frac{2 \mu Z}{\alpha \hbar^{2}}, \Lambda^{2}=\frac{2 \mu l(l+1)}{\alpha \hbar^{2}}
$$

By comparing Eq. (1) with Eq. (48), we obtain the following

$$
\tilde{\tau}(x)=0, \sigma(x)=x, \tilde{\sigma}(x)=-\varepsilon^{2} x^{2}-\delta^{2} x-\Lambda^{2}
$$

The function $\pi(x)$ is obtained by using $\operatorname{Eqs}(7)$ and (50) as

$$
\pi(x)=\frac{1}{2} \pm \sqrt{\varepsilon^{2} x^{2}+\left(\delta^{2}+k\right) x+\left(\Lambda^{2}+\frac{1}{4}\right)}
$$

The expression in the square root in $\mathrm{Eq}(51)$ must be the polynomial based on NU method and $k$ can be determined as

$$
k=-\delta^{2} \pm \sqrt{\varepsilon^{2}+4 \Lambda^{2} \varepsilon^{2}}
$$

Consequently, for each value of $k$, the function $\pi(s)$ is obtained as

$$
\pi(x)=\frac{1}{2} \pm \begin{cases}\varepsilon x+\frac{1}{2} \sqrt{1+4 \Lambda^{2}} & \text { for } k=-\delta^{2}+\sqrt{\varepsilon^{2}+4 \Lambda^{2} \varepsilon^{2}} \\ \varepsilon x-\frac{1}{2} \sqrt{1+4 \Lambda^{2}} & \text { for } k=-\delta^{2}-\sqrt{\varepsilon^{2}+4 \Lambda^{2} \varepsilon^{2}}\end{cases}
$$

Negative derivative of $\tau(s)$ is obtained by selecting the physical values of $\pi(x)$ and $k$ as

$$
\pi(x)=\frac{1}{2}-\left(\varepsilon x-\frac{1}{2} \sqrt{1+4 \Lambda^{2}}\right)
$$

and

$$
k=-\delta^{2}-\sqrt{\varepsilon^{2}+4 \Lambda^{2} \varepsilon^{2}}
$$

Substituting Eq (54) in Eq. (10) gives the function $\tau(x)$ as

$$
\begin{aligned}
& \tau(x)=1-2 \varepsilon x+\sqrt{1+4 \Lambda^{2}} \\
& \tau^{\prime}(x)=-2 \varepsilon
\end{aligned}
$$

Using Eqs. (8) and (9) the following expression for $\lambda$ are obtained respectively as

$$
\begin{gathered}
\lambda=-\delta^{2}-\sqrt{\varepsilon^{2}+4 \Lambda^{2} \varepsilon^{2}}-\varepsilon \\
\lambda=\lambda_{n}=2 n \varepsilon
\end{gathered}
$$

Equating Eq. (57) to Eq. (58) and making useof Eq. (49), we obtain the energy eigenvalue as 


$$
\mathrm{E}_{n}=-\frac{2 \mu z^{2}}{\alpha^{2} h^{2}}\left[1+2 n+\sqrt{1+\frac{8 \mu}{\alpha \hbar^{2}} l(l+1)}\right]^{2}
$$

Using Eq. (6) the weight function $\rho(x)$ is found to be

$$
\rho(x)=x^{\sqrt{1+4 \Lambda^{2}}} e^{-2 \varepsilon x}
$$

Using weight function $\rho(x)$, we now obtain $y_{n}(x)$ as

$$
y_{n}(x)=C_{n} x^{-\sqrt{1+4 \Lambda^{2}}} e^{2 \varepsilon x} \frac{d^{n}}{d x^{n}}\left[x^{n+\sqrt{1+4 \Lambda^{2}}} e^{-2 \varepsilon x}\right]
$$

The other past of the wave function $\phi(x)$ is obtained as

$$
\phi(x)=x^{\frac{1}{2}\left(1+\sqrt{1+4 \Lambda^{2}}\right)} e^{-\varepsilon x}
$$

Combining Eqs. (61) and (62), the wave function for this system is obtained as

$$
\psi(x)=C_{n} x^{\frac{1}{2}\left(1+\sqrt{1+4 \Lambda^{2}}\right)} e^{-\varepsilon x} L_{n}^{\sqrt{1+4 \Lambda^{2}}}(x)
$$

where $L_{n}^{\sqrt{1+4 \Lambda^{2}}}(x)$ are associated Laguerre polynomials

\section{SOLUTIONS OF SOLVABLE PT SYMMETRIC POTENTIAL}

\subsection{PT SYMMETRIC ROSEN-MORSE II POTENTIAL}

In Eq. (11) we set $\alpha \rightarrow i \alpha$, , then it turns into

$V(x)=-\frac{a(a+1)}{\cos ^{2} \alpha x}+2 i \gamma \tan \alpha x$

If we write the potential in the Schrödinger equation and using transformation as $s=\tan \alpha x$, the energy spectrum is obtained as

$$
\begin{aligned}
& E_{n}=\frac{\alpha^{2} \hbar^{2}}{4 \mu A^{* 2}}\left(2 A^{* 3}+D^{*}+F^{*}-\frac{8 \mu^{2} \gamma^{2} A^{*}}{\alpha^{4} \hbar^{4}}\right) \pm \frac{\alpha^{2} \hbar^{2}}{4 \mu A^{* 2}}\left[\left(2 A^{* 3}+D^{*}+F^{*}-\frac{8 \mu^{2} \gamma^{2} A^{*}}{\alpha^{4} \hbar^{4}}\right)^{2}-4 A^{* 2}\right. \\
& \left.\left\{\left(A^{* 2}-\frac{4 \mu^{2} \gamma^{2}}{\alpha^{4} \hbar^{4}}\right)^{2}+G^{* 2}-\frac{8 A^{*} \mu G^{*}}{\alpha^{2} \hbar^{2}}+\frac{16 \mu^{2} \gamma^{2} A^{* 2}}{\alpha^{4} \hbar^{4}}\right\}\right]^{\frac{1}{2}}
\end{aligned}
$$


where

$$
\begin{aligned}
& A^{*}=-\left[\frac{2 \mu a(a+1)}{a^{2} \hbar^{2}}+2 n+2 n(n-1)\right] \\
& D^{*}=\left[\frac{4 \mu a(a+1)}{a^{2} \hbar^{2}}+4 n+2 n(n-1)^{2}-\frac{16 \mu^{2} \gamma^{2}}{\alpha^{4} \hbar^{4}}\right]\left(n+\frac{1}{2}\right)^{2} \\
& F^{*}=\left[\frac{16 \mu^{2} \gamma^{2}}{\alpha^{4} \hbar^{4}}\left(\frac{1}{2}+n(n+1)-\frac{2 \mu a(a+1)}{a^{2} \hbar^{2}}\right)\right] \\
& G^{*}=-\frac{8 \mu \gamma}{\alpha^{2} \hbar^{2}}\left(n+\frac{1}{2}\right)^{2}
\end{aligned}
$$

The corresponding wave function is

$$
\psi(x)=B_{n}[1-i \tan (\alpha x)]^{\frac{i v}{2}}[1+i \tan (\alpha x)]^{i \xi} P_{n}^{(i \xi+i v)}(i \tan (\alpha x))
$$

\subsubsection{PT symmetric scarf II potential}

If we use $\alpha \rightarrow i \alpha$ in Eq. (30), then it turns into

$$
V(x)=\frac{-1}{\cos ^{2} \alpha x}\left[\left(\frac{\gamma+\beta}{2}\right)^{2}+\left(\frac{\gamma-\beta}{2}\right)^{2}-\frac{1}{4}\right]+\frac{i \sin \alpha x}{\cos ^{2} \alpha x} \frac{\beta+\gamma}{2} \frac{\beta-\gamma}{2}
$$

Writing this potential in the Schrödinger equation and using the transformation $s=\sin a x$, the energy spectrum is obtained as

$$
\begin{aligned}
& E_{n}=-\frac{1}{2}\left(\beta^{2}-\gamma^{2}\right)-\frac{\alpha^{2} \hbar^{2}}{2 \mu}\left[\frac{1}{8} \sqrt{\left(1-\frac{2 \mu}{\alpha^{2} \hbar^{2}}\left(\beta^{2}-\gamma^{2}\right)\right)^{2}+\frac{64 \mu^{2}}{\alpha^{4} \hbar^{4}}\left(\beta^{2}-\gamma^{2}-\frac{1}{2}\right)^{2}}-\right. \\
& \frac{3}{8}-2 n-n(n-1)+\left(\frac{1+2 n}{4}\right)\left(\sqrt{1-\frac{2 \mu}{\alpha^{2} \hbar^{2}}\left(\beta^{2}-\gamma^{2}\right)-\frac{i 8}{\alpha^{2} \hbar^{2}}\left(\beta^{2}-\gamma^{2}-\frac{1}{2}\right)-}\right. \\
& \left.\left.\sqrt{1-\frac{2 \mu}{\alpha^{2} \hbar^{2}}\left(\beta^{2}-\gamma^{2}\right)+\frac{i 8}{\alpha^{2} \hbar^{2}}\left(\beta^{2}+\gamma^{2}-\frac{1}{2}\right)}\right)\right]
\end{aligned}
$$

and the corresponding wave function as

$$
\psi(x)=B_{n}[1-\sin (\alpha x)]^{\frac{1}{4}\left(1+\xi^{\prime}\right)}[1+\sin (\alpha x)]^{\frac{1}{4}\left(1-v^{\prime}\right)} P_{n}^{\left(\frac{1}{z^{\prime}},-\frac{1}{2} v^{\prime}\right)}(-\sin (\alpha x))(69)
$$




\subsubsection{PT Coulomb potential}

By setting $\alpha \rightarrow i \alpha$ in Eq. (47), then the potential turns to

$$
V(x)=\frac{-i z}{\alpha x}-\frac{i l(l+1)}{\alpha x^{z}}=-i\left[\frac{z}{\alpha x}+\frac{l(l+1)}{\alpha x^{z}}\right]
$$

The energy spectrum of this potential is obtained as

$$
E_{n}=\frac{2 \mu z^{2}}{\alpha^{z} \hbar^{z}}\left[1+2 n+\sqrt{1-\frac{i 8 \mu l(l+1)}{\alpha \hbar^{z}}}\right]^{-2}
$$

The corresponding wave function is

$$
\psi(x)=C_{n} x^{\frac{1}{2}\left(1+\sqrt{1-i 4 \Lambda^{2}}\right)} e^{-\varepsilon x} L_{n}^{\sqrt{1-i 4 \Lambda^{2}}}(x)
$$

\section{CONCLUSIONS}

We solved the Schrodinger equation for non-complex and complex potentials using the Nikiforov-Uvarov method. The non-PT symmetric Rosen Morse II and Coulomb potentials have real energy spectra except Scarf II that remain purely real and imaginary. In the case of the PT symmetric the energy spectra of Rosen Morse II potential is purely real while that of Scarf II and Coulomb potentials are complex. However, the complex version of the potentials show that the PT symmetric complex potentials have real energy spectra for only Rosen-Morse II while other potentials have complex energy spectra. Thus, PT symmetry is not the necessary condition for the reality of the energy spectrum [17]. Therefore, it is worth extending the approach to the solution of other interactingproblems. Our results are not only interesting for the pure theoretical physicists but also for experimental physicists because they are exact.

\section{ACKNOWLEDGEMENT}

The authors are thankful to Dr. Akpan N. Ikot of the Theoretical Physics Group, Department of Physics, University of Port Harcourt, Nigeria for his invaluable suggestions which led to the improved standard of this paper. We also acknowledge the reviewer for the positive comment .

\section{REFERENCES}

[1] C. M. Bender and B. Boettcher (1998). Real spectra in non Hermitian Hamiltonians having PT symmetry, Phys. Lett. 80,4243.

[2] C. M. Bender(2007). Making sense of non-Hermitian Hamiltonians, Rep. Prog. Phys 70, 947.

[3] G. Levai (2011). Asymptotic properties of solvable PT-symmetric potential, Int. J. Theor. Phys 50, 997.

[4] S. M. Ikhdair and R. Sever(2005). Polynomial solution of PT/ non-PT- symmetric and nonHermitian Generalized Woods-Saxon potential via Nikiforov-Uvarov method. arXiv:quant$\mathrm{ph} / 0507272$

[5] Mario Catagnino, Jorge Gueron and Adolfo Ordonez(2002). Global time asymmetry as a consequence of a wave packets theorem, J. Math. Phys 43, 705. 
[6] C. M. Bender, S. Boettcher and P. N. Meisinger (1999). PT-Symmetric Quantum Mechanics, J. Math Phys 40, 2201.

[7] O. Yesiltas(2006). PT/ non-PT Symmetric and non-Hermitian Poschl-Teller-like solvable potentials via Nikiforov-Uvarov method. Phys Scr. 75,41.

[8] Z. Ahmed (2001). Pseudo-Hermiticity of Hamiltonians under imaginary shift of the coordinate: real spectrum of complex potentials, Phys Lett A 290, 19.

[9] T. V. A. Fityo (2002). A new class of non-Hermitian Hamiltonians with real spectra, J. Phys A $35,5893$.

[10] C. M. Bender, G. V. Dunne and P. N. Meisinger(1999). Complex periodic potential with real band spectra, Phys Lett A 252.

[11] E. Delabaere and F. Bham (1998). Eigenvalues of complex Hamiltonians with PT-symmetry, Phys Lett A 250, 25.

[12] C. M. Bender, K. A. Milton and V. M. Savage (2000). Solution of Schwinger-Dyson equations for PT-Symmetric quantum field theory, Phys Rev. D62, 085001.

[13] C. M. Bender, F. Cooper, P. N. Meisinger and M. V. Savage(1999). Variational Ansatz for PTSymmetric Quantum Mechanics, Phys Lett. A 259,229.

[14] B. Bagchi and C. Quesne(2002). Non-Hermiltonians with real and complex eigenvalues in a Liealgebraic framework, Phys.Lett. A 300, 18.

[15] A. F. Nikiforov and V. B. Uvarov(1998). Special functions of mathematical Physics (Birhauser, Basel).

[16] C. Berkdemi, A. Berdemie and R. Sever(2006). Symmetrical approach to the exact solution of the Dirac equation for a deformed form of the Woods-Saxon potential, J. Phys. A: Math Gen 399, 13455.

[17] Z. A. Ping, Q. W. Chao and L. Y. Wen(2009). Approximate solution of the Schrödinger equation for the Eckart potential and its parity-time-symmetric version including centrifugal term, Chin. Phys. Lett 26(10), 100302. 